\title{
Synthesis Method and FPGA Realization of Quasi-barker Codes
}

\author{
Ivan Tsmots \\ Department of Automated Control Systems \\ Lviv Polytechnic National University \\ Lviv, Ukraine
}

\author{
Oleg Riznyk \\ Department of Publishing Information Technologies \\ Lviv Polytechnic National University \\ Lviv, Ukraine
}

\author{
Vasyl Rabyk \\ Department of RadioPhysics and Computer Technologies \\ Ivan Franko National University of Lviv \\ Lviv, Ukraine
}

\begin{abstract}
The paper considers the method of obtaining quasi-Barker codes based on numerical ruler-bundles. Their advantages, basic parameters and areas of application are given. The algorithm is implemented on FPGA EP3C16F484N6 of Altera firm. The functional diagram of the DS-SS system using quasi-barker codes is presented, Quasi-Barker codes generator, implemented in VHDL language and its simulation in frequency domain was described.
\end{abstract}

Index Terms-DS-SS; Barker code; quasi-barker code; autocorrelation function; FPGA; hardware implementation.

\section{INTRODUCTION}

The choice of pseudorandom code sequence in the radio engineering system of a information transmission is very important, since its parameters depend on its noise immunity, sensitivity [1]. For the same length of the code sequence the system parameters may be different.

Among the large variety of signals particularly interesting are signals Barker signals - the signals with low level sidelobes of the autocorrelation function (ACF). These signals ensure the achievement of the high value of the main ACF lobe under the condition of a low level of sidelobes. Barker signals are conveniently explored and constructed using numeric sequences, the elements of which are code symbols 1 and -1 .

Research results $[5,10]$ showed that there are no Barker signals with an odd number of positions greater than 13 for which the values of ACF do not exceed one (with the exception of the mainlobe). Among the well-known Barker signals, the maximum ratio of the mainlobe to other lobes is 13 , while the ratio of quasi-barker code signals found to be equal to 14 or more [6]. Therefore, there is a sense to continue research in this direction.

So far, there is no universal algorithm that delivers an acceptable quality of signal processing in all radar tasks $[9,10]$.
In this regard, the modern radar station should contain a constantly replenished set of algorithms and signals, which when combined in a pair of signal-algorithm can provide a solution to a certain range of tasks $[4,7]$.

This work is devoted to the development of methods of synthesis noise codes using quasi-barker sequences for encoding and decoding data; development of a simulation model for the synthesis of noise code by criteria autocorrelation function, sequence length and a number of detected and corrected errors; implementation of quasi-barker sequences obtained on FPGA. The subject of research is the ACF function models of barker-like codes and methods of finding it.

\section{BARKER CODES}

Barker codes are used in the spread spectrum communication system. These codes are well suited for systems Direct Sequence-Spread Spectrum (DS -SS) as they have several advantages over other pseudo-Noise (PN) codes.

To expand the spectrum in a narrowband signal in DS-SS systems, in each of its transmitted bits, the sequence of chips is embedded, which is called a noisy code. Each of the chips can be represented as a sequence of rectangular pulses of duration in $\mathrm{n}$ times less than the duration of the information bit. The spectrum expansion for two information bits is depicted in Fig. 1. The pseudo-noise code here uses the Barker code length $N=7$. This picture contains: $d_{t}-$ information signal (two bits), $T_{b}-$ period of each bit, $b c_{t}-$ Barker code, $T_{c}-$ period of each chip, $t x_{t}-$ - a transformed signal generated by transmitting signals $d_{t}$ and $b c_{t}$ through an XOR element with a negation. 


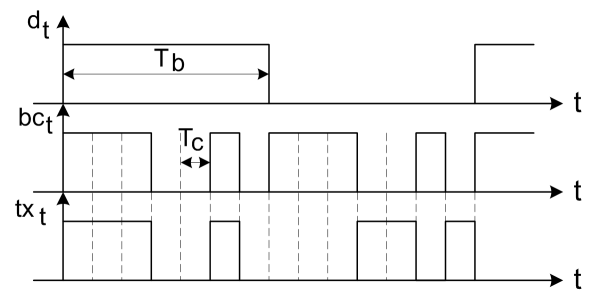

Fig. 1. Expantion of Barker code with length $N=7$

The functional diagram of the DS-SS system is shown in Fig. 2. In transmitter the transformed signal $t x_{t}$ is modulated. This is often done using the Binary Phase Shift Keying (BPSK) method. In the receiver, the modulated signal is restored by the BPSK demodulation method.

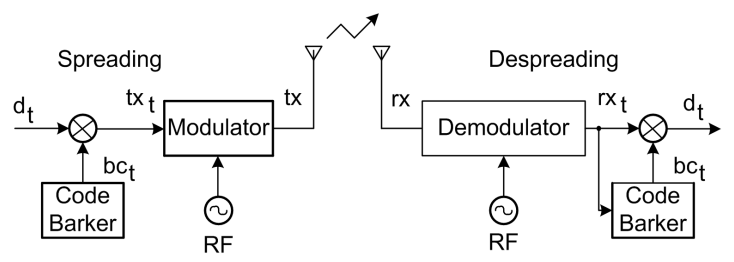

Fig. 2. Functional scheme of the system DS-SS

One of the main blocks of each DS-SS system is the PNsequence generator (Barker code).

The Barker sequence is a series consisting of $N$ elements of $a_{j}$ for $1 \leq j \leq N$, which have values +1 and -1 and alternate so that the condition is fulfilled:

$$
\left|\sum_{j=1}^{N-i} a_{j} a_{j+i}\right| \leq 1
$$

where $1 \leq i<N$.

Barker's sequences have a minimal level of sidelobe ACF, and therefore they are suitable for optimal reception. Known Barker sequences have a length $2 \leq N \leq 13$.

ACF Barker sequence is a finite discrete sequence, which is formed by a convolutional operation over the sequence and its own copy:

$$
R_{j}=\sum_{i=1}^{N-j} a_{i} a_{i+j}^{*}
$$

where $i$ the discrete index of the shift between the sequence and its copy (in time), and the designation indicates the complex conjugate value. The autocorrelation function is symmetric with respect to the mainlobe, which follows from its general properties.

ACF coefficient module for zero displacement $j=0$ was called the Mainlobe level - ML. The mainlobe level has the highest value compared to other coefficients of the ACF and is equal to its length $N$.
Peak sidelobe level - PSL is defined as the maximum absolute value among the coefficients of the autocorrelation function for a non-zero shift $1 \leq j<N$ :

$$
\mathrm{PSL}=\max \left\{|| \sum_{i=1}^{N-j} a_{i} a_{i+j} \mid\right\} .
$$

The level of sidelobes for Barker codes does not exceed 1. The peak sidelobe level ratio to its mainlobe, which is measured in decibels became widespread:

$$
\operatorname{PSLR}=20 \log _{10}\left(\frac{\mathrm{PSL}}{\mathrm{ML}}\right)
$$

As an example, let's consider Barker code with length $\mathrm{N}=13(+1,+1,+1,+1,+1,-1,-1,+1,+1,-1,+1,-1,+1)$, for which $\mathrm{PSL}=1, \mathrm{ML}=13 \mathrm{i} \mathrm{PSLR}=-22.279 \mathrm{~dB}$. His $\mathrm{ACF}$ is shown in Fig. 3.

\section{Barker code autocorrelation}

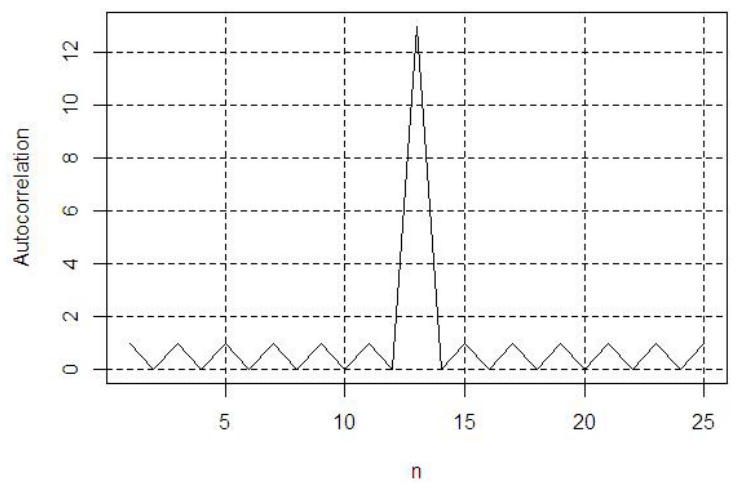

Fig. 3. Autocorrelation of Barker sequence length $\mathrm{N}=13$

\section{ALGORITHM FOR THE SYNTHESIS OF QUASI-BARKER CODES}

In the general case, the simplicity of the numerical ruler bundle (NRB) of the order of $N$ in the sequence of $N$ numbers, is called the sequence $K_{N}=\left(k_{1}, k_{2}, \ldots, k_{N}\right)$, in which sum take the values of all $L_{N}$ numbers starting from a given number. In a simpler version, these amounts exhaust the values of the numbers of the natural series $1,2, \ldots, L_{N}[5]$.

If a series of successive values of the mentioned sums begins with $a$, then the total sum of all numbers in this series is defined as [8]:

$$
S_{N}=\frac{K(K+2 a-1)}{2}
$$

Dependence between the number of $K$ methods for the implementation of sums on $N$ - sequence, multiplicity $R$ and sum $L_{N}$ all numbers are expressed by the formula [6]: 


$$
\left(L_{N}-1\right) R=K-1 \text {. }
$$

An important parameter of a system using quasi-barker codes is the processing gain, which shows the measure of the improvement of the signal-to-noise ratio when converting the noise signal received by the receiver into the required information signal. This procedure is called despreading. According to the classic definition, $B_{O}$ is equal:

$$
B_{0}=10 \log _{10}\left(\frac{\mathrm{C}_{k}}{\mathrm{C}_{\mathrm{i}}}\right)
$$

where $C_{k}$ - the frequency of receiving pseudorandom sequence chips (Chip/sec), $C_{i}$ - the speed of transmission of information (bit/sec). For a system with $C_{i}=1 \mathrm{Mbit} / \mathrm{sec}$ and $C_{k}=13 \mathrm{MChip} / \mathrm{sec}$ (each bit of information is encoded in a pseudo-random sequence of 13 bits) the winning on processing will be $B_{0}=11.14 \mathrm{~dB}$. In this case, the efficiency of the information transmission system will be maintained if the useful input signal is reduced by $11,14 \mathrm{~dB}$.

Synthesis quasi-barker codes algorithm based on numerical ruler bundles with a minimum value of the autocorrelation function of a discrete signal is considered in [2] and is as follows:

- choose the NRB variant of the given order $N$ of the required length $L_{N}$ with multiplicity $R$ [] using random movement algorithm (with $2<N<12$ ), asymmetric branching algorithm (with $12<N<18$ ) f,j NRB construction algorithm based on the ideal ring bundles (with $18 \leq N$ );

- building of $L_{N^{-}}$position code $\mu_{i}, i=1,2, \ldots, L_{N}$ with a onelevel periodic function of autocorrelation based on the chosen version of the NRB $\left(k_{1}, k_{2}, \ldots, k_{N}\right)$, where the $N$ lines of the code with sequence numbers $x_{l}, l=1,2, \ldots$, are placed with the characters "1", and in the remaining $L_{N}-N$ positions - with the characters "-1".

Sequence numbers $x_{l}$ are determined by the formula:

$$
x_{l} \equiv 1+\sum_{i=1}^{l} k_{i} \cdot\left(\bmod L_{N}\right)
$$

The resulting sequence is a pulse sequence with the property of "no more than R-matches" and the minimum values of the autocorrelation function. By choosing another variant of the NRB with such parameters (if it exists), we can receive other pulse sequences with the property "no more than R-matches" and the minimum value of the autocorrelation function.

Quasi-barker codes in length $L_{N}=14 \ldots 40$ are described in [5]. For each of the lengths $L_{N}$ of these quasi-barker codes, the level of sidelobes of the normalized correlation function is minimal. The calculation of the unique codes for each $L_{N}$ length was performed with the help of NRB.
As an example, let's consider the building of quasi-barker code according to the above algorithm for $N=12, L_{N}=21$, $R=7$ [5]. To do this one need to take a few steps:

- of the four existing variants of the simple shortest NRB of the order $N=12$, constructed by the algorithm of selective displacements [6], we select the first variant of the NRB: $(1,1,1,1,1.2,4,2,1,4,1,2)$;

- we construct a sequence in which the length of the code is $L_{N}=21$.

To do this in twelve positions $(N=12)$, calculated by the formula (4), we place the characters " 1 ", and fill the remaining positions with the characters "-1":

$$
1,1,1,1,1,1,-1,1,-1,-1,-1,1,-1,1,1,-1,-1,-1,1,1,-1 \text {. }
$$

We received the quasi-barker code for which PSLR $=$ 20.42 , its ACF is depicted in fig. 4. From this figure, we see that PSL $=2$, the level of the mainlobe is equal to $\mathrm{ML}=21$. The number of options with a minimum level of AKF - 12.

\section{Quasi-Barker code autocorrelation - N=21}

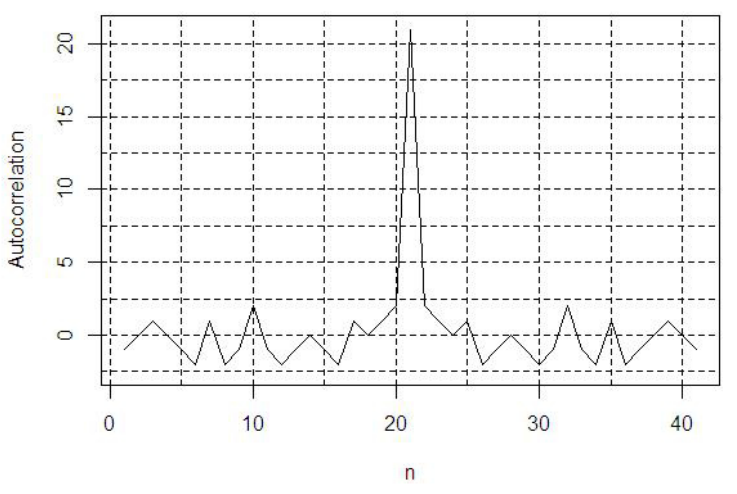

Fig. 4. Autocorrelation of Barker sequence length $\mathrm{N}=21$

\section{REALIZATION OF BARKER CODES ON FPGA}

For the implementation of Barker codes and quasi-barker codes, FPGA EP3C16F484N6 of the family Cyclone III of the firm Altera, which is part of the stand DE0, was used. [6]. The development was carried out in the programming language of the VHDL hardware in the Quartus II development environment using the development environment libraries. FPGA allows you to implement parallel computing with a large data, have the sufficiently large number of hardware on their crystal. So the FPGA EP3C16F484 includes 15484 logical elements (LEs), 56 blocks of M9K, 56 multipliers $18 \times 18$, a large number of implemented IP cores [7]. FPGA supports high-speed interfaces with external storage. LE is the smallest element of logic in the architecture of the Cyclone III family. Each LE has four inputs, a four-way conversion table (LUT), a register, and an output logic [7].

The main component of the quasi-barker code generator is the successive-parallel shift register. The scheme of generating a quasi-barker code with dimensionality $N=28$ is depicted in Fig. 5 


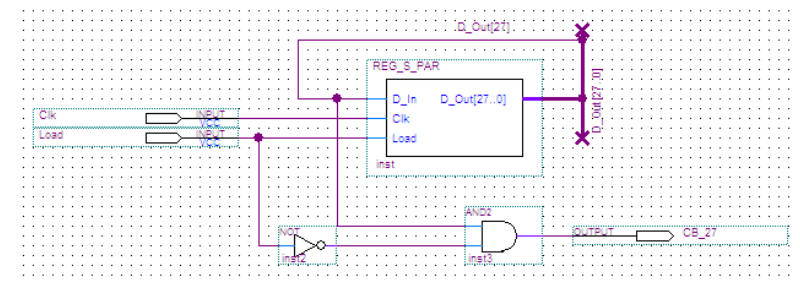

Fig. 5. Appearance of the quasi-barker code generation scheme $(N=28)$

This register contains the value 0x9FB2B94 (1001111110110010101110010100B) as initial value for offset.

The character of the generator of quasi-barker codes with length $N=28$ (CB_28) is shown in Fig. 6.

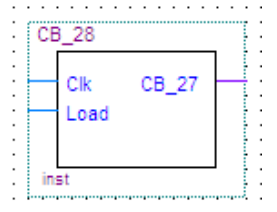

Fig. 6. The appearance of the symbol CB_28

Inputs of quasi-barker code generator with dimensionality $N=28: \mathrm{Clk}$ - the input of synchronization in the register of a sequential parallel shift; Load (active signal level "1") - initial load signal data in the register. The output of the generator CB 28 is a quasi-barker code with dimension $N=28$. During each Clk synchronization pulse, a one-bit offset to the left of the data array D_Out [27...0] is done and record the input value $D$ In register $\bar{R} E G S$ PAR into junior $D$ Out [0]. Time diagrams of the device $\overline{C B} 28$ shown in Fig. 7.

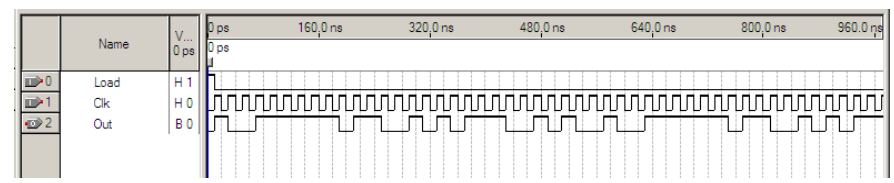

Fig. 7. Time diagrams of the quasi-barker code with dimensionality $N=28$

Similarly, it is possible to implement a quasi-barker code generator of arbitrary dimension $N=14 \ldots 40$. This will change the hardware resources needed for this. So, for the implementation of quasi-barker code generator with dimension $\mathrm{N}=28$ it is necessary 29 (from 15408) logical elements, 28 registers and 3 (out of 347) FPGA EP3C16F484 pins.

\section{CONCLUSION}

The algorithm of quasi-barker synthesis and their codes with dimensionality $N=14 . .40$ is developed. Autocorrelation functions for these codes are investigated. Implemented quasibarker codes on FPGA EP3C16F484 family firm Altera Cyclone III and of their work in the time domain was performed.

\section{REFERENCES}

[1] C. R. Lakshmi, D. Trivikramarao, S. Subhani and V. S. Ghali, "Barker coded thermal wave imaging for anomaly detection," 2018 Conference on Signal Processing And Communication Engineering Systems (SPACES), Vijayawada, India, 2018, pp. 198-201. doi: 10.1109/SPACES.2018.8316345.

[2] Cyclone III Device Handbook. http://www.altera.com/literature/hb/cyc3/ cyc3_ciii51001.pdf.

[3] DE0 User Manual. Development and Education Board. http:// esca.korea.ac.kr/teaching/FPGA_boards/DE0/DE0_User_Manual.pdf

[4] M. Kellman, F. Rivest, A. Pechacek, L. Sohn and M. Lustig, "BarkerCoded node-pore resistive pulse sensing with built-in coincidence correction," 2017 IEEE International Conference on Acoustics, Speech and Signal Processing (ICASSP), New Orleans, LA, 2017, pp. 10531057. doi: 10.1109/ICASSP.2017.7952317.

[5] O. Riznyk, B. Balych and I. Yurchak, "A synthesis of barker sequences is by means of numerical bundles," 2017 14th International Conference The Experience of Designing and Application of CAD Systems in Microelectronics (CADSM), Lviv, 2017, pp. 82-84. doi: 10.1109/CADSM.2017.7916090.

[6] O. Riznyk, O. Povshuk, Y. Kynash and I. Yurchak, "Composing method of anti-interference codes based on non-equidistant structures," 2017 XIIIth International Conference on Perspective Technologies and Methods in MEMS Design (MEMSTECH), Lviv, 2017, pp. 15-17. doi: 10.1109/MEMSTECH.2017.7937522.

[7] Pilsu Kim, Eunji Jung, Sua Bae, Kangsik Kim and Tai-kyong Song, "Barker-sequence-modulated golay coded excitation technique for ultrasound imaging," 2016 IEEE International Ultrasonics Symposium (IUS), Tours, 2016, pp. 1-4. doi: 10.1109/ULTSYM.2016.7728737.

[8] Riznyk Oleg, Kynash Y., P. Oleksandr and B. Bohdan, "Information technologies of optimization of structures of the systems are on the basis of combinatorics methods," 2017 12th International Scientific and Technical Conference on Computer Sciences and Information Technologies (CSIT), Lviv, 2017, pp. 232-235. doi: 10.1109/STCCSIT.2017.8098776.

[9] S. M. Omar, F. Kassem, R. Mitri, H. Hijazi and M. Saleh, "A novel barker code algorithm for resolving range ambiguity in high PRF radars," 2015 European Radar Conference (EuRAD), Paris, 2015, pp. 81-84. doi: 10.1109/EuRAD.2015.7346242.

[10] Siti Julia Rosli1, Hasliza Rahim, Ruzelita Ngadiran, K. N. Abdul Rani, Muhammad Imran Ahmad and Wee, "Design of Binary Coded Pulse Trains with Good Autocorrelation Properties for Radar Communications," 2018 MATEC Web of Conferences, doi: $10.1051 /$ matecconf $/ 201815006016$. 